\title{
Modelo de decisão sobre o uso de preservativos: uma regressão logística multinomial.
}

\section{Decision model about condom use: a multinomial logistic regression.}

\section{Modelo de decisión sobre la utilización de condones: una regresión logística multinomial.}

\author{
Isis Milane Batista de Lima ${ }^{1}$ \\ Ana Eloisa Cruz de Oliveira ${ }^{2}$ \\ Josemberg Moura de Andrade ${ }^{3}$ \\ Hemílio Fernandes Campos Coêlho ${ }^{4}$ \\ Kaline Silva Lima ${ }^{5}$
}

RESUMO: O presente trabalho apresenta uma estratégia de estudo para auxiliar o processo de tomada de decisão sobre a autoeficácia no uso do preservativo por meio da regressão logística multinomial. Os dados analisados são referentes a uma amostra de 1260 brasileiros, dos quais 36\% afirmaram sempre usar camisinha. De acordo com o modelo de decisão considerado, as variáveis analisadas revelaram que a decisão sobre o uso do preservativo é influenciada pela confiança que o indivíduo sente no sucesso do seu uso.

Palavras-chave: Autoeficácia, preservativos, modelo logístico.

\footnotetext{
ABSTRACT: This work aims to present a study strategy to assist the decision making process about self-efficacy on condom use by multinomial logistic regression. The data analyzed refers to a sample composed of 1260 Brazilians, of which $36 \%$ reported always using condoms. According to the decision model considered, the variables indicated that the decision on condom use is modulated by the confidence that the individual feels about the success of its use.

Keywords: Self-Efficacy, condoms, logistic model.

1Bacharel em Estatística pela Universidade Federal da ParaÃba e doutoranda do Programa de Pós Graduação em Modelos de Decisão e Saúde. Campus I - Jardim Cidade Universitária. Departamento de Estatística. João Pessoa-PB Brasil. E-mail: isismilane@hotmail.com

2 Enfermeira pela Universidade Federal da Paraíba e doutoranda do Programa de Pós Graduaação em Modelos de Decisão e Saúde. E-mail: elocruz17@hotmail.com

3 Professor da Universidade Federal da Paraíba. E-mail: josemberg.andrade@gmail.com

4 Professor da Universidade Federal da Paraíba. E-mail: hemilio@gmail.com

5 Psicóloga pela Universidade Federal da Paraíba. E-mail: kaline.s.lima@hotmail.com
} 
RESUMEN: Este trabajo presenta una estrategia de estudio para ayudar en el proceso de toma de decisiones, respecto a la autoeficacia en el uso de condones, mediante la regresión logística multinomial. Los datos analizados se refieren a una muestra de 1260 brasileños, de los cuales el 36\% reportó usar siempre condones. De acuerdo con el modelo de decisión considerado, las variables indican que la decisión sobre el uso del condón se ve influenciada por la confianza que el individuo tiene sobre el éxito de su uso.

Palabras clave: autoeficacia, condones, modelo logístico.

\section{INTRODUÇÃO}

A autoeficácia é a medida de quão competente o indivíduo se sente para fazer algo, controlando seus resultados. Está relacionada com realizações alcançadas, autoconfiança, autoestima e até mesmo, por meio de manipulações sutis, pode afetar o comportamento sexual. No entanto, nem sempre a autoeficácia dispõe de pontos positivos ou benefícios, sendo capaz de aumentar a vulnerabilidade individual através do otimismo ilusório ou irrealista que pode gerar. Por isso, o realismo pode ser a melhor opção diante dos perigos do otimismo irrealista ${ }^{1}$.

O termo foi derivado da Teoria da Aprendizagem Social de Bandura ${ }^{2}$, na qual o autor propôs que a autoeficácia é um mediador importante de comportamento e definiu conceitualmente como 'um julgamento da própria capacidade para realizar um determinado nível de desempenho'. A teoria ressalta ainda o indivíduo que 'não está preocupado com as habilidades que se tem, mas com julgamentos sobre o que se pode fazer com as habilidades que possui'.

A Teoria da autoeficácia tem considerável importância nos estudos comportamentais relacionados à saúde, incluindo a autoprevenção da transmissão sexual do HIV e outras doenças sexualmente transmissíveis, além da ocorrência da gravidez indesejável. Tem, portanto, implicações importantes para a previsão e redução de comportamentos de risco à saúde dos indivíduos 3 .

O preservativo é um recurso disponível a homens e mulheres, que oferece um meio seguro, econômico e eficaz que atende à dupla função de prevenção de gravidez indesejada e de doenças sexualmente transmissíveis (DST), quando é utilizado de forma consistente e adequado. Além disso, é relativamente barato, com pouca ou nenhuma restrição ao uso. Pode ser utilizado com segurança, sem exigir qualquer habilidade específica ${ }^{4}$.

Mesmo com todas as facilidades apresentadas pelo preservativo, indivíduos de ambos os sexos ainda apresentam resistências explícitas ou veladas ao seu uso. Este fato está diretamente relacionado ao conhecimento, à atitude e à prática que cada indivíduo possui, estabelecendo uma mudança na vida sexual para aqueles que não têm o hábito de utilizá-lo ${ }^{5}$.

Devido ao caráter incurável da Síndrome da Imunodeficiência Adquirida (SIDA/AIDS), evitar infecções por HIV é um dos maiores desafios de saúde pública, em boa parte do mundo. Nesse 
contexto, a redução de danos e a prevenção se destacaram como estratégias fundamentais para o enfrentamento da epidemia. No entanto, a estratégia de prevenção é prejudicada pelo baixo uso de preservativos, especialmente por pessoas que vivem em áreas onde há epidemia de HIV/AIDS, mesmo com os preservativos prontamente disponíveis ${ }^{6}$.

A disseminação da educação sobre as práticas sexuais das pessoas torna-se cada vez mais relevante, uma vez que o desenvolvimento de conhecimentos e habilidades pessoais consiste em uma das estratégias de promoção da saúde. Ações neste âmbito podem ser capazes de promover autonomia e corresponsabilidade no cuidado com o próprio corpo e na quebra da cadeia de transmissão de doenças sexualmente transmissíveis, fazendo com que os indivíduos conheçam várias opções de cuidado e conduzam práticas favoráveis à saúde

Quando a discussão de assuntos relacionados com o uso de preservativo, a prevenção de DST e a sexualidade não constituem uma prática comum e existe simultaneamente uma sujeição às normas do grupo - comunidade - ou às normas culturais nos mais variados aspectos, poderá surgir com maior facilidade à aceitação de crenças pouco adequadas em relação ao preservativo, que posteriormente poderão vir a influenciar os comportamentos protetores. Neste sentido, a percepção de autoeficácia ou as crenças poderão ser variáveis importantes, na medida em que podem influenciar o comportamento das pessoas no que diz respeito ao uso do preservativo ${ }^{8}$.

Além de fatores como conhecimento e acesso, a autoeficácia no uso de preservativos tem se mostrado fortemente associada ao comportamento de cada indivíduo frente ao uso do mesmo, sendo um importante conceito a ser estudado na área da saúde, motivando o desenvolvimento do estudo em questão, cujo objetivo visa contribuir com a discussão acerca da tomada de decisão sobre o uso do preservativo e sua relação com a autoeficácia.

\section{METODOLOGIA}

Trata-se de um estudo exploratório descritivo com abordagem quantitativa, que contou com uma amostra 1.260 pessoas das cinco regiões do Brasil: Nordeste (37,3\%), Sudeste (25\%), Centrooeste (16\%), Norte $(8,7 \%)$ e Sul (12,6\%). Tal amostra foi não probabilística e de conveniência. Os sujeitos foram contatados via redes sociais, bem como por correio eletrônico para responder ao questionário. Nesse caso, os resultados aqui encontrados não podem ser generalizados. Procurouse obter um quantitativo similar de sujeitos por região geográfica, embora isso não tenha sido alcançado. O número de sujeitos foi definido em função das exigências das análises estatísticas e psicométricas realizadas em outras etapas da pesquisa, tais como Teoria de Resposta ao Item, análise fatorial confirmatória, entre outras.

Além de um questionário sociodemográfico, no qual foram perguntados aos sujeitos dados como a cidade e o estado onde residem, além da renda familiar, idade, estado civil, nível de escolaridade, orientação sexual, entre outros, o instrumento foi composto pela escala derivada da 
pesquisa Condom Use Self-Efficacy Scale-Ghana 9 . Os autores defendem o conceito de autoeficácia proposto por Bandura ${ }^{2}$, cujas influências gerais estão ligadas à capacidade de julgamentos do que se pode fazer com suas próprias habilidades. Também enfatizam que na autoeficácia se reflete o nível de confiança de uma pessoa na sua capacidade para controlar o ambiente ${ }^{3,9}$. A escala avalia a autoeficácia no uso de preservativo, ou seja, a percepção do indivíduo de usar preservativos. Essa escala possui 14 itens distribuídos numa escala Likert com os seguintes extremos: 1 = 'Discordo Totalmente' e 5 'Concordo Totalmente'. O conjunto de itens se divide em quatro subescalas: Habilidade (colocar um preservativo em si mesmo ou no outro); a Assertividade (capacidade de convencer um parceiro a usar preservativo); Prazer e drogas (capacidade de usar preservativos quando se está sob a influência de alguma substância); e Doenças sexualmente transmissíveis (receio que o parceiro pense que tem uma doença sexualmente transmissível).

Os itens da Escala Condom Use Self-Efficacy Scale - Ghana (CUSES-G) foram traduzidos e adaptados para a cultura brasileira através de uma validação semântica ${ }^{10}$. Após aprovação do comitê de ética do Hospital Universitário Lauro Wanderley, com o nº 17887013. 1. 0000. 5183, realizou-se a aplicação dos questionários através de um formulário online, demandando a resposta individual dos participantes, que foram orientados a não se identificarem no questionário, assegurando o anonimato de sua participação. Foi indicado que poderiam deixar o estudo a qualquer momento sem penalização, garantindo o caráter voluntário da participação. Todos concordaram com um Termo de Consentimento Livre e Esclarecido exposto previamente, no qual se apresentou os objetivos da pesquisa e as informações sobre os pesquisadores, momento esse no qual foram expostas também as instruções de cada instrumento.

A regressão logística é uma técnica estatística que tem como objetivo produzir, a partir de um conjunto de observações, um modelo que permita a predição de valores tomados por uma variável categórica, frequentemente binária, a partir de uma série de variáveis explicativas contínuas e/ou binárias. No presente estudo foi empregado um tipo de regressão logística denominada multinomial, a qual permite a análise das variáveis explicativas com a variável resposta em seus vários níveis, cuja variável dependente possui mais de duas classes mutuamente exclusivas ${ }^{11}$.

No modelo de regressão logística, considerando uma situação em que a variável resposta poderá assumir apenas duas possibilidades de valores, '0' ou ' 1 ', supõe-se que o modelo tenha a seguinte forma:

$$
Y_{i}=x_{i}^{T} \beta+\varepsilon_{i} Y_{i}=x_{i}^{T} \beta+\varepsilon_{i},
$$

No qual:

$x_{i}^{T}=\left[1, x_{i 1}, x_{i 2}, \ldots, x_{i p}\right],\left[\beta_{0}, \beta_{1}, \ldots, \beta_{p}\right]$,

e a variável resposta $Y_{i} Y_{i}$ com distribuição Bernoulli assume os valores 0 ou 1 com as respectivas probabilidades

Tempus, actas de saúde colet, Brasília, 10(2), 67-80, jun, 2016.

ISSN 1982-8829 


$$
\pi_{i}=P(Y i=1) \pi_{i}=P(Y i=1) \text { e } 1-\pi_{i}=P(Y i=0) \cdot 1-\pi_{i}=P(Y i=0) .
$$

Supondo que $E\left(\varepsilon_{i}\right)=0 E\left(\varepsilon_{i}\right)=0 \mathrm{E}\left(\varepsilon_{\mathrm{i}}\right)=0_{E}\left(\varepsilon_{i}\right)=0$, o valor esperado da variável resposta é dada por

$$
E\left(Y_{i}\right)=\left[1 \times\left(\pi_{i}\right)\right]+\left[0 \times\left(1-\pi_{i}\right)\right]=\pi_{i} E\left(Y_{i}\right)=\left[1 \times\left(\pi_{i}\right)\right]+\left[0 \times\left(1-\pi_{i}\right)\right]=\pi_{i},
$$

Isto implica que

$$
E\left(Y_{i}\right)=x_{i}^{T} \beta=\pi_{i} E\left(Y_{i}\right)=x_{i}^{T} \beta=\pi_{i}
$$

Portanto, o valor esperado para variável aleatória $Y Y$, dada pela função resposta

$$
E\left(Y_{i}\right)=x_{i}^{T} \beta E\left(Y_{i}\right)=x_{i}^{T} \beta
$$

é a probabilidade da variável resposta $Y Y$ assumir o valor $1^{12}$.

Entretanto, existem alguns problemas com o modelo de regressão expresso em (1). Note que se a resposta for binária, o erro $\varepsilon_{i} \varepsilon_{i}$ poderá assumir apenas dois valores

$$
\begin{aligned}
& \stackrel{\bullet}{\bullet} \varepsilon_{i}=1-x_{i}^{T} \beta \varepsilon_{i}=1-x_{i}^{T} \beta \varepsilon_{\mathrm{i}}=1-\mathrm{x}_{\mathrm{i}}^{\mathrm{T}} \beta \quad \varepsilon_{i}=1-x_{i}^{T} \beta, \text { quando } Y_{i}=1 \\
& Y_{i}=1, \mathrm{y}_{\mathrm{i}}=1 y_{i}=1 \\
& \text { - } \varepsilon_{i}=-x_{i}^{T} \beta \varepsilon_{i}=-x_{i}^{T} \beta, \text { quando } Y_{i}=0 Y_{i}=0 . \mathrm{y}_{\mathrm{i}}=0_{\mathrm{y}_{\mathrm{i}}}=0
\end{aligned}
$$

Consequentemente, os erros deste modelo não terão a possibilidade de serem normais e a variância não será constante. Finalmente, há uma restrição da função resposta, pois

$$
0 \leq E\left(Y_{i}\right)=\pi_{i} \leq 10 \leq E\left(Y_{i}\right)=\pi_{i} \leq 1
$$

esta restrição pode causar sérios problemas com a escolha da função linear para variável resposta, como expresso na expressão (1), já que neste caso o modelo poderia ser ajustado com valores preditivos fora do intervalo $[0,1]$. Geralmente, quando a variável resposta é binária, há evidências empíricas de que a forma da função resposta é não linear. Neste caso, emprega-se uma transformação na expressão (1) que passa a se chamar função resposta logística, segundo os autores anteriores, sob a forma

$$
E\left(Y_{i}\right)=\frac{\exp \left(x_{i}^{T} \beta\right)}{1+\exp \left(x_{i}^{T} \beta\right)} E\left(Y_{i}\right)=\frac{\exp \left(x_{i}^{T} \beta\right)}{1+\exp \left(x_{i}^{T} \beta\right)},
$$

ou equivalente

$$
E\left(Y_{i}\right)=\frac{1}{1+\exp \left(-x_{i}^{T} \beta\right)} E\left(Y_{i}\right)=\frac{1}{1+\exp \left(-x_{i}^{T} \beta\right)} .
$$


A função logística pode ser facilmente linearizada pela transformação logitlogit, definida por:

$\eta_{i}=\ln \left(\frac{\pi_{i}}{1-\pi_{i}}\right) \eta_{i}=\ln \left(\frac{\pi_{i}}{1-\pi_{i}}\right)$

Onde $\eta_{i}=x_{i}^{T} \beta \eta_{i}=x_{i}^{T} \beta$ é denominado preditor linear.

No modelo de regressão logística multinomial considera-se uma coleção de $r+1$ $r+1$ variáveis independentes, denotadas por $X=\left(X_{0}, X_{1}, \ldots, X_{r}\right) X=\left(X_{0}, X_{1}, \ldots, X_{r}\right)$, onde $x=\left(x_{0}, x_{1}, \ldots, x_{r}\right) x=\left(x_{0}, x_{1}, \ldots, x_{r}\right) \operatorname{com} x_{0}=1 x_{0}=1$ e $Y Y$ uma variável aleatória nominal que pode assumir os níveis $0,1, \ldots, q 0,1, \ldots, q$, e comparando-se $Y=k Y=k \operatorname{com} Y=0 Y=0$, para $k \in\{1,2, \ldots, \mathrm{q}\} k \in\{1,2, \ldots, \mathrm{q}\}$. O valor zero então é denominado categoria de referência ${ }^{(13)}$.

Denota-se a funcão lo gitlogit como sendo

$$
\begin{aligned}
& g_{k}=\ln \left\lceil\frac{\mathbb{P}(Y=k \mid x)}{\mathbb{P}(Y=0 \mid x)}\right\rceil \\
& =\beta_{k 0} x_{k 0}+\beta_{k 1} x_{k 1}+\ldots+\beta_{k r} x_{k r} \\
& =x^{T} \beta_{k}=x^{T} \beta_{k} .
\end{aligned}
$$

Adotando-se $n n$ observações independentes de $Y Y$, associadas aos valores de $x_{i}=\left(x_{i 0}, \ldots, x_{i r}\right)$ $x_{i}=\left(x_{i 0}, \ldots, x_{i r}\right)$, para $i \in\{1,2, \ldots, \mathrm{n}\} i \in\{1,2, \ldots, \mathrm{n}\}$, o logit, expresso em (2), é dado por

$$
g_{k n}=\beta_{k 0} x_{n 0}+\beta_{k 1} x_{n 1}+\ldots+\beta_{k r} x_{n r}+\varepsilon_{n} g_{k n}=\beta_{k 0} x_{n 0}+\beta_{k 1} x_{n 1}+\ldots+\beta_{k r} x_{n r}+\varepsilon_{n},
$$

sendo $x_{i 0}=1 x_{i 0}=1, i \in\{1,2, \ldots, \mathrm{n}\} i \in\{1,2, \ldots, \mathrm{n}\}$ e os erros, $\varepsilon_{i} \varepsilon_{i}$, seguem as seguintes suposições:

$$
\begin{gathered}
E\left(\varepsilon_{i}\right)=0 ; \operatorname{Var}\left(\varepsilon_{i}\right)=\operatorname{Var}\left(Y_{i}\right) ; \operatorname{Cov}\left(\varepsilon_{i}, \varepsilon_{l}\right)=0, \text { sei } \neq l \\
E\left(\varepsilon_{i}\right)=0 ; \operatorname{Var}\left(\varepsilon_{i}\right)=\operatorname{Var}\left(Y_{i}\right) ; \operatorname{Cov}\left(\varepsilon_{i}, \varepsilon_{l}\right)=0, \text { sei } \neq l .
\end{gathered}
$$

Deste modo, o modelo de regressão logística multinomial é definido como:

$$
\pi_{k i}(x)=\frac{\exp \left(g_{k i}\right)}{1+\exp \left(g_{k i}\right)} \pi_{k i}(x)=\frac{\exp \left(g_{k i}\right)}{1+\exp \left(g_{k i}\right)},
$$

no qual $g_{k i} g_{k i}$ é obtido em (2), $x_{i j} x_{i j}$ é constante conhecida, $\beta_{k j} \beta_{k j}$ é um parâmetro desconhecido e assumindo as suposições do erro $\varepsilon_{i} \varepsilon_{i}$.

O intervalo de confiança $100(1-\alpha) \% 100(1-\alpha) \%$ de $\beta_{k j} \beta_{k j}$ é dado por:

$$
\left[\widehat{\beta_{k j}} \pm z_{\frac{\alpha}{2}} \overline{S E} \bar{E}\left(\widehat{\beta_{k j}}\right)\right]\left[\widehat{\beta_{k J}} \pm z_{\frac{\alpha}{2}} \overline{S E} \bar{E}\left(\widehat{\beta_{k j}}\right)\right]
$$

onde $z \underline{\alpha} \underline{\alpha} \underline{\alpha}$ é o quantil de uma normal padrão e $\left.\left.\bar{S} \bar{E}\left(\widehat{\beta_{k_{1}}}\right)\right] \bar{S} \bar{E}\left(\widehat{\beta_{k_{\jmath}}}\right)\right]$ representa o estimador do desvio pad̃ã̃o de $\overline{\beta_{k_{j}}} \cdot \widehat{\beta_{k_{j}}}$. 
A estatística de Wald, dado uma matriz de informação $I(\hat{\beta}) I(\hat{\beta})$, é baseada na estatística $W=\hat{\beta}^{T}[I(\hat{\beta})] \hat{\beta}^{T}$

Sabe-se que sob $H_{0}: \beta=0 H_{0}: \beta=0$ a estatística $\mathrm{W}$ possui distribuição Qui-quadrado com $q(r+1) q(r+1)$ graus de liberdade ${ }^{13}$.

O intervalo de confiança $100(1-\alpha) \% 100(1-\alpha) \%$ de $\pi_{k}(x) \pi_{k}(x)$ é dado por:

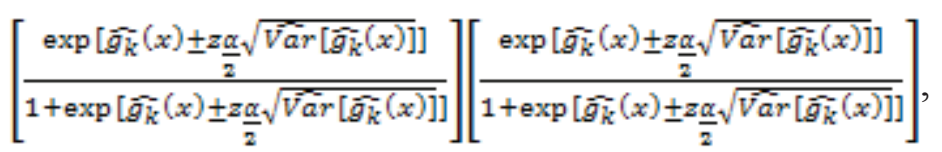

onde $\quad z \underline{\alpha} z \underline{\alpha}$ é o quantil de uma normal padrão e $\widehat{\operatorname{Var}}\left[\widehat{g_{k}}(x) \widehat{\operatorname{Var}}\left[\widehat{g_{k}}(x)\right]=x^{\prime} \widehat{\operatorname{Var}}\left[\widehat{\beta_{k}}(x)\right]\right.$ $=x^{\prime} \overline{\operatorname{Var}}\left[\widehat{\beta_{k}}(x)\right]$.

Para avaliar a qualidade do ajuste do modelo de regressão logística multinomial, será utilizado neste estudo o Pseudo $R^{2} R^{2}$ de Nagelkerke, que explica o poder total das variáveis independentes do modelo ${ }^{14}$.

\section{RESULTADOS E DISCUSSÃO}

Buscando avaliar a autoeficácia no uso de preservativos, foram estudadas 1260 pessoas, as quais responderam a um questionário composto pela Escala de Autoeficácia no Uso de Preservativos, que possui quatro fatores, habilidade, assertividade, prazer e drogas e DST. A amostra foi composta em sua maioria por pessoas do sexo feminino $(65,6 \%)$, com idades que variaram entre 18 e 62 anos $(\mathrm{M}=23,9 ; \mathrm{DP}=6,5)$, heterossexuais $(86,5 \%)$, solteiros $(73,6 \%)$, com ensino superior incompleto (69\%), e possuem renda de 1 a 3 salários mínimos (25,7\%). Em relação ao uso do preservativo, $36,3 \%$ disseram usar sempre e $56 \%$ usam camisinha mesmo tendo parceiro fixo.

Após o período do estudo verificou-se o grau de autoeficácia no uso do preservativo para cada indivíduo, por meio da técnica de regressão logística multinomial, aplicada através da versão 3.1.3 do software $\mathrm{R}^{15}$. As seguintes variáveis foram definidas para o estudo:

- Variável resposta - Uso do preservativo, que é uma variável policotômica e assume os valores 1, 2, 3, 4 e 5, respectivamente, sempre usa, usa frequentemente, às vezes usa, raramente usa e nunca usa.

- Variáveis dependentes - As variáveis dependentes analisadas encontram-se apresentadas na Tabela a seguir: 
Quadro 1 - Descrição das variáveis utilizadas nos fatores da Escala.

\begin{tabular}{|c|c|}
\hline Variável & Descrição \\
\hline EAUP1 & Eu me sentiria envergonhado em botar um preservativo em mim ou meu $\backslash$ minha parceiro(a) \\
\hline EAUP2 & $\begin{array}{l}\text { Sinto-me confiante de que poderia colocar ou remover tranquilamente um preservativo quando } \\
\text { tenho relações. }\end{array}$ \\
\hline EAUP3 & $\begin{array}{l}\text { Sinto-me confiante em minha capacidade de colocar um preservativo em mim ou meu } \backslash \text { minha } \\
\text { parceiro (a) durante as preliminares. }\end{array}$ \\
\hline EAUP4 & $\begin{array}{l}\text { Sinto-me confiante de que posso usar um preservativo com meu } \backslash \text { minha parceiro(a) sem 'atrapalhar } \\
\text { o momento'. }\end{array}$ \\
\hline EAUP5 & Sinto-me confiante de que posso usar um preservativo com sucesso. \\
\hline EAUP6 & $\begin{array}{l}\text { Sinto-me confiante em minha capacidade para discutir o uso do preservativo com qualquer } \\
\text { parceiro(a) que eu possa ter. }\end{array}$ \\
\hline EAUP7 & $\begin{array}{l}\text { Sinto-me confiante em minha capacidade de sugerir o uso de preservativo com um(a) novo(a) } \\
\text { parceiro(a). }\end{array}$ \\
\hline EAUP8 & $\begin{array}{l}\text { Sinto-me confiante de que poderia sugerir o uso de preservativo sem que meu parceiro se sinta } \\
\text { 'doente'. }\end{array}$ \\
\hline EAUP9 & $\begin{array}{l}\text { Sinto-me confiante de que posso utilizar um preservativo durante uma relação sem diminuir o } \\
\text { prazer sexual. }\end{array}$ \\
\hline EAUP10 & $\begin{array}{l}\text { Sinto-me confiante de que posso me lembrar do uso do preservativo, mesmo após ter ingerido } \\
\text { bebidas alcoólicas. }\end{array}$ \\
\hline EAUP11 & Sinto-me confiante de que posso me lembrar do uso preservativo, mesmo após ter usado drogas. \\
\hline EAUP12 & $\begin{array}{l}\text { Não me sentiria confiante sugerindo o uso de preservativo a um(a) novo(a) parceiro(a), porque } \\
\text { sentiria medo dele(a) pensar que já tive experiências homossexuais. }\end{array}$ \\
\hline EAUP13 & $\begin{array}{l}\text { Não me sentiria confiante sugerindo o uso de preservativo a um(a) novo(a) parceiro(a), porque } \\
\text { sentiria medo dele(a) pensar que tenho uma doença sexualmente transmissível. }\end{array}$ \\
\hline EAUP14 & $\begin{array}{l}\text { Não me sentiria confiante sugerindo o uso de preservativo a um(a) novo(a) parceiro(a), porque } \\
\text { sentiria medo dele(a) pensar que já tive uma doença sexualmente transmissível. }\end{array}$ \\
\hline
\end{tabular}

Fonte: Tradução própria.

A Figura 1 apresenta a frequência com que os indivíduos fazem o uso do preservativo. É possível observar que apenas 3\% dos entrevistados afirmaram nunca ter usado a camisinha, 17\% afirmaram que às vezes usam, 18\% afirmaram usam raramente, 26\% afirmaram que usam frequentemente e, por fim, 36\% afirmaram sempre usar o preservativo.

Figura 1 - Distribuição percentual do Uso do preservativo entre os entrevistados.

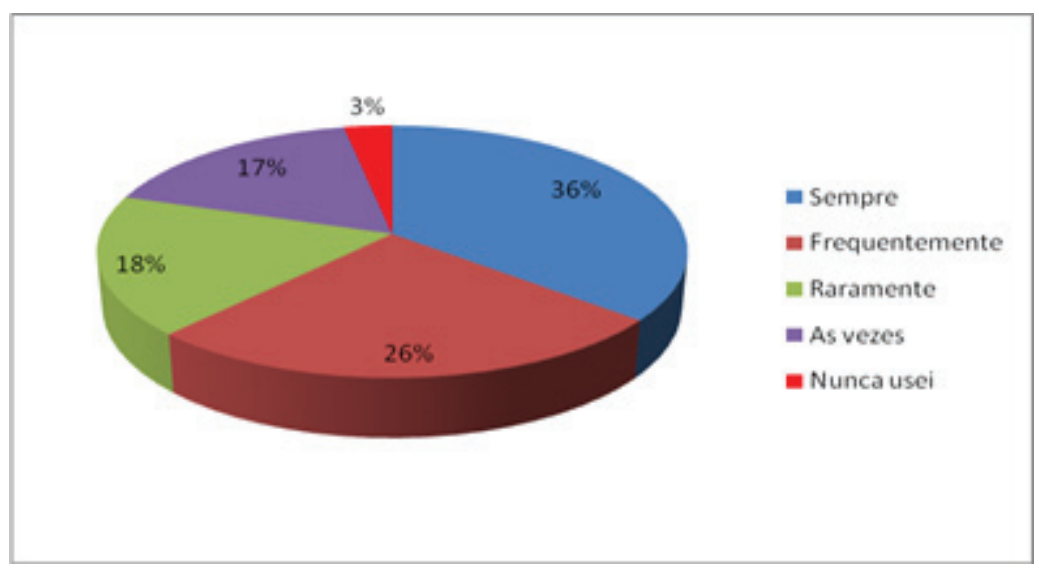

Fonte: Elaboração própria. 
Assumindo o modelo de regressão logística multinomial, uma das categorias da variável resposta deve ser designada como categoria de referência e as demais serão comparadas com esta categoria. Como a escolha é arbitraria, neste caso, assume-se como categoria de referência a variável 'nunca usei'.

O pseudo $R^{2} R^{2}$ de Nagelkerke apresentou valor de $12 \%$, fornecendo evidência de que o modelo de regressão logística multinomial captou uma pequena parte da influência sofrida pela variável dependente com relação aos fatores adotados nesta analise. Deve-se levar em consideração de que a medida é um pseudo $R^{2} R^{2}$, podendo não refletir integralmente o poder explicativo total das variáveis independentes.

De acordo com a Tabela 2, pode-se dizer que não há evidências de que os coeficientes que descrevem o modelo são nulos, pois os níveis de significância apresentados nos testes são menores ou iguais a 5\%, que foi o nível adotado para esse estudo. E ainda, os fatores considerados importantes, isto é, os que foram significativos para o modelo foram EAUP5 - "Sinto-me confiante de que posso usar um preservativo com sucesso", EAUP9 - "Sinto-me confiante de que posso utilizar um preservativo durante uma relação sem diminuir o prazer sexual" e EAUP10 - "Sintome confiante de que posso lembrar-me do uso do preservativo mesmo após ter ingerido bebidas alcoólicas", todos $\operatorname{com} p<0,05 p<0,05$.

Tabela 2 - Teste da contribuição das variáveis ao modelo

\begin{tabular}{c|c|c|c}
\hline Efeito & Qui-Quadrado & Grau de liberdade & P valor \\
\hline EAUP5 & 10,128 & 4 & 0,03 \\
\hline EAUP9 & 36,115 & 4 & 0,00 \\
\hline EAUP10 & 9,435 & 4 & 0,05 \\
\hline
\end{tabular}

Fonte: Elaboração própria.

A Tabela 3 apresenta os resultados do modelo que mostra evidência de que o uso de camisinha é influenciado pelos níveis estabelecidos: 'sempre uso', 'uso frequentemente', 'às vezes uso', 'raramente uso' e 'nunca usei', e pela variável EAUP5, que foi considerada a variável mais significativa. Como a categoria de referência é 'nunca usei' então quer dizer que o uso da camisinha é explicado pela influência da confiabilidade que o indivíduo tem no sucesso do preservativo. Um intervalo de confiança representa uma amplitude de valores que tem alta probabilidade de conter o verdadeiro valor da população. Assim sendo, pode-se dizer que com 95\% de confiança a variação em uma unidade no uso de camisinha acarreta, para a função logitlogit, uma variação, entre 1, 179 e 2,378 , para quem sempre usa, entre $[1,175 ; 2,399]$ para quem usa frequentemente, $[1,272$; $2,687]$ para quem usa às vezes e por fim, $[1,087 ; 2,265]$ para quem raramente usa.

A coluna ExpExp indica as razões de chance nas variáveis independentes e dependentes, isto é, o aumento do uso de camisinha acarreta acréscimo na função logitlogit, visto que seu valor 
é maior que um. Quando o indivíduo confia no sucesso do uso do preservativo, então tem-se um aumento do seu uso em $67,5 \%$, em relação à categoria dos indivíduos que afirmam nunca utilizar o preservativo. Para os que usam frequentemente essa chance é bem próxima, chegando a $67,9 \%$ do uso a mais, em relação à categoria dos que afirmam nunca utilizarem. Para os que afirmam fazer uso às vezes, a chance de uso aumenta ainda mais: $84 \%$ a mais do que quando eles confiam no sucesso do uso do preservativo. Para os que raramente usam o preservativo a chance é diminuída para $56,7 \%$ quando se confia no seu sucesso.

Tabela 3 - Parâmetros estimados para o Modelo Logístico Multinomial

\begin{tabular}{|c|c|c|c|c|c|c|c|}
\hline \multirow[b]{2}{*}{ Coeficiente } & \multirow[t]{2}{*}{ B } & \multirow[t]{2}{*}{ Wald } & \multirow[t]{2}{*}{$\begin{array}{l}\text { Grau de } \\
\text { liberdade }\end{array}$} & \multirow[t]{2}{*}{ P valor } & \multirow[t]{2}{*}{$\operatorname{Exp}(B)$} & \multicolumn{2}{|c|}{$\begin{array}{l}\text { Intervalo de Confiança } \\
\text { de } 95 \% \text { para } \operatorname{Exp}(B)\end{array}$} \\
\hline & & & & & & $\begin{array}{l}\text { Limite } \\
\text { Inferior }\end{array}$ & $\begin{array}{l}\text { Limite } \\
\text { Superior }\end{array}$ \\
\hline Sempre uso & 0,516 & 8,309 & 1 & 0,004 & 1,675 & 1,179 & 2,378 \\
\hline Uso frequentemente & 0,518 & 8,089 & 1 & 0,004 & 1,679 & 1,175 & 2,399 \\
\hline Ás vezes uso & 0,614 & 10,367 & 1 & 0,001 & 1,849 & 1,272 & 2,687 \\
\hline Raramente uso & 0,450 & 5,775 & 1 & 0,016 & 1,569 & 1,087 & 2,265 \\
\hline
\end{tabular}

Fonte: Elaboração própria. Nota: Categoria de Referência Nunca usei.

Ainda conforme a Tabela 3 os dados da estatística de Wald foram significativos, com isso, rejeita-se a hipótese nula de que os coeficientes são iguais a zero e de que a variável independente selecionada não produz efeito sobre a variável dependente. Portanto, o modelo de regressão logística multinomial foi bem ajustado e a variável decisória, o uso de preservativo, é influenciado pela confiança que o indivíduo possui no sucesso do seu uso. Ou seja, a decisão em usar preservativos é influenciada pelo nível de autoeficácia dos indivíduos para realizar esta tarefa de forma adequada. Sendo assim, torna-se importante a realização de intervenções no âmbito da saúde e educação que estimulem o desenvolvimento da autoeficácia no uso de preservativos da população, a fim de aumentar a escolha por este método de proteção e diminuir as vulnerabilidades às doenças sexualmente transmissíveis e gravidezes indesejadas.

A amostra avaliada no estudo, apesar de ter captado uma pequena parte da influência sofrida pela variável dependente, com relação aos fatores adotados nesta análise, apresentou-se de forma significativa ao final do estudo, mostrando que a variável significativa mais relevante ao modelo foi à relacionada à confiança das pessoas. Deste modo, observa-se a importância de se confiar não só no parceiro, mas também na escolha de um método preventivo às doenças e ainda à gravidez indesejada.

As relações entre os casais são complexas e possuem diversos fatores relacionados ao seu comportamento sexual e às suas práticas. A escolha pelo uso da camisinha está relacionada ao conhecimento inadequado, às práticas inseguras e atitudes que distorcem a realidade dos indivíduos analisados, gerando mudanças em seus planos de vida conjugais ou individuais. Um dos motivos 
que levam os casais a não usarem preservativos consiste no medo de que o parceiro pense que ele/ ela tem alguma doença sexualmente transmissível ${ }^{16}$.

Em termos de diferenças de gênero, o machismo encontrado na cultura brasileira ainda influencia nas atitudes relacionadas à sexualidade e aos seus valores, impondo certa pressão social nas negociações e discussões acerca do uso de preservativo, nem sempre levantadas de forma saudável pelos homens, pois, estes abordam a questão com preconceito, causando constrangimento à sua parceira. Em consequência, a parceira acaba cedendo, não simplesmente pela confiança, mas por estar afetivamente envolvida, possuir um relacionamento duradouro e/ou até pelo medo de perder o parceiro. Com isso, os esforços de prevenção do HIV em mulheres só terão sucesso efetivo na dependência da conscientização feminina e da mudança de comportamento masculino, com o aumento do uso do preservativo ${ }^{17}$.

A relação com a prevenção do HIV e uso do preservativo acaba sendo construída ainda na adolescência, com o início da vida sexual. As relações sexuais sem penetração podem ser preditas pelas atitudes positivas em relação ao preservativo e que as relações sexuais com penetração podem acontecer por aspectos como adaptação pessoal e escolar ${ }^{18}$, destacando-se ainda a importância da idade da primeira relação sexual como determinante para comportamentos sexuais arriscados.

No entanto, uma negativa imagem dos preservativos produzidos por experiências pessoais mal sucedidas, pela má reputação e por mitos, sendo associados algumas vezes à sujeira, ao sexo ilícito e à infidelidade, podem surgir não só no período da adolescência, como em qualquer momento ao longo da vida de cada pessoa. Nessa perspectiva, para que a tomada de decisão seja favorável ao uso do preservativo, alguns dos grandes desafios brasileiros consiste em reforçar a qualidade da assistência do Sistema Único de Saúde (SUS), promover a conscientização a respeito do risco de relações sexuais desprotegidas, e garantir a sustentação do comprometimento entre as esferas políticas e governamentais em prover recursos materiais e profissionais capacitados e motivados, para trabalhar de forma contínua essas questões como sexualidade, conhecimento e confiança, abrangendo indivíduos de todas as idades e, assim, promover o aumento na adesão do preservativo, principalmente de forma consciente dos benefícios gerados por essa decisão ${ }^{19}$.

\section{CONSIDERAÇÕES FINAIS}

A rápida globalização que está ocorrendo traz consigo uma maior preocupação acerca da saúde sexual dos indivíduos, e alguns dos motivos que influenciam essa realidade é o fato do comportamento sexual não ser algo estático e da multiplicidade de fatores que se encontram relacionados a isso, pois implicam a necessidade de avaliações periódicas ${ }^{20}$.

As mudanças no âmbito dos comportamentos sexuais foram capazes de gerar importante impacto sobre as doenças sexualmente transmissíveis e especialmente a HIV/AIDS, na década de 1980, fazendo com que os métodos de prevenção tivessem uma nova ênfase. Dentre as diferentes formas de profilaxia das DST, várias agências de saúde, governamentais ou não, têm focado, com maior empenho, a propagação do uso do preservativo, também conhecido como camisinha, levando em consideração o seu elevado grau de proteção documentado frente ao risco de aquisição de DST, 
inclusive o HIV/AIDS ${ }^{4}$.

Os países iniciaram o marketing social de preservativos, em sincronia com os esforços globais de planejamento familiar em desenvolvimento, e foi dramaticamente expandido como uma resposta rápida para a AIDS. Com isso, houve um esforço coordenado para garantir um fornecimento estável de preservativos de qualidade a nível local nos países e o uso desse preservativo como forma de proteção mútua dos parceiros, solicitando a inclusão do homem nas decisões da vida sexual e reprodutiva do casal ${ }^{21}$.

$\mathrm{Na}$ abordagem significativa do uso do preservativo, torna-se relevante considerar a Teoria da Autoeficácia, uma vez que essa levanta estudos comportamentais relacionados à saúde, considerando tais implicações importantes para a previsão e redução de comportamentos de risco à saúde dos indivíduos. Relacionando a autoeficácia e a tomada de decisão frente ao uso do preservativo, no estudo em questão, o modelo de regressão logística clássica não foi possível ser utilizado, já que a variável resposta usa camisinha não foi anteriormente categorizada de forma binária, tendo 0 como não usa e 1 como usa o preservativo, pois isso acarretaria perda de informação para a sua análise estatística. Assim sendo, o método utilizado para analisar as variáveis apresentadas, de forma adequada, foi a regressão logística multinomial, que tornou possível uma análise mais precisa dos resultados. Este modelo possui vantagens que o modelo logístico clássico não considera, mantendo todos os níveis da variável resposta.

Para essa pesquisa, por meio da regressão logística multinomial, conclui-se que a confiança do indivíduo foi a variável mais relevante para tal análise, ou seja, exerce importante influência no momento de decisão do uso do preservativo. Dessa forma, é possível perceber que para aumentar a adesão ao preservativo, não basta simplesmente intensificar a sua distribuição, mas sim investir simultaneamente no aumento de discussões sobre o tema, promovendo mais abertura na mídia para lidar com falta de conscientização das pessoas, sensibilizando-as de forma a considerar aspectos como a questão de confiança que se tem em relação ao seu parceiro, como em si mesmo.

A partir do estudo também se notou a ausência de artigos que abordem a questão da confiança em relação ao uso do preservativo na literatura existente, sendo necessário um maior investimento em produções científicas sobre a temática e a abordagem levantada, para que, assim, contribua-se ainda mais com as práticas no que diz respeito às decisões tomadas pela população frente à sua saúde sexual e reprodutiva.

\section{REFERÊNCIAS BIBLIOGRÁFICAS}

1. Myers DG. Psicologia Social. 6 edição. Rio de Janeiro: LTC; 2000.

2. Bandura A. Social Foundations of Thought and Actions: A Social Cognitive Theory. New Jersey: Prentice - Hall; 1986.

3. Forsyt AD, Carey MP. Measuring self-efficacy in the context of HIV risk reduction: research hallenges and recommendations. Health Psychology. 1998; 17(6): 559. 
4. Aragão JCS, Lopes C de S, Bastos FI. Comportamento sexual de estudantes de um curso de medicina do Rio de Janeiro. Rev. bras. educ. med. 2011; 35(3): 334-40.

5. Sousa LB de, Cunha D de FF, Ximenes LB, Pinheiro AKB, Vieira NFC. Conhecimentos, atitudes e prática de mulheres acerca do uso do preservativo. Rev. enferm. UERJ. Jan-mar 2011; 19(1): 146-51.

6. Smith KP, Watkins SC. Perceptions of risk and strategies for prevention: responses to HIV/ AIDS in rural Malawi. Soc Sci Med. 2005; 60(3): 649-60.

7. Carreno I, Costa JSD da. Uso de preservativos nas relações sexuais: estudo de base populacional. Rev. Saúde Pública. 2006; 40(4): 720-6.

8. Ribeiro J, Leal I, Dias E. Autoeficácia e crenças em mulheres jovens - O caso específico do preservativo/kamisinha (um estudo comparativo). $3^{\circ}$ Congresso Nacional de Psicologia da Saúde; 2000; Lisboa.

9. Asante KW, Doku PN. Cultural adaptation of the Condom Use Self Efficacy Scale (CUSES) in Ghana.BMC Public Health. 2010.

10. Lima KS, Andrade JM, Sá LA, Pernambuco JM, Gaudêncio CA. Evidências de validade de construto e confiabilidade da escala de autoeficácia no uso de preservativos. VI Congresso Brasileiro de Avaliação Psicológica e IX Congresso Iberoamericano de Diagnóstico Y Evaluación Psicológica; 2013; Maceió.

11. Silva A, Brito J, Gaspar PL. Análise probabilística da condição de degradação dos revestimentos pétreos (fixados diretamente ao suporte). Teoria e Prática na Engenharia Civil. Lisboa. Nov 2011; 18: 49-61.

12. Montgomery DC, Peck EA, Vinnig GG. Introduction to linear regression analysis. $3^{\mathrm{a}}$ edição. New York: Wiley-Interscience; 2001.

13. Figueira CV. Modelos de regressão logística. Universidade Federal do Rio Grande do Sul. Porto Alegre; 2006.

14. Smith TJ, Mckenna CMA. Comparison of Logistic Regression Pseudo R2 Indices. Multiple Linear Regression Viewpoints. 2013; 39(2).

15. R: A language and environment for statistical computing. R Foundation for Statistical Computing, Vienna, Austria.URL http://www.R-project.org/ .

16. Ballester R, Gil-Ilario MD, Ruiz-Palomino E, García CG. Autoeficacia en la prevención sexual del Sida: la influencia del gênero. Anales de psicologia.enero 2013; 29(1): 76-82.

17. Pires MRM, Balieiro CRB. Representações sobre DST/AIDS e o uso de preservativo nas 
relações sexuais entre homens heterossexuais. Saúde \& Transformação Social / Health \& Social Change. Universidade Federal de Santa Catarina. 2012; 3(3): 89-104.

18. Castro A, Bérmudez MP, Buela-Casal G, Madrid J. Variables psicossociales que median em el debut sexual de adolescentes em España. Rev. Latino-americana de Psicologia. 2011; 43(1): 83-94.

19. Laroque MF et al. Sexualidade do idoso: comportamento para a prevenção de DST/AIDS. Rev. Gaúcha Enferm. 2011; 32(4): 774-80.

20. Vega-Briones G, Jaramillo-Cardona MC. Percepciones y actitudes de los hombres de la frontera norte de México en relación con el uso de condones, práctica de la vasectomía y chequeos de próstata. Rev. Gerenc. Polit. Salud, enero-junio 2010; 9(18): 50-77.

21. Sweat MD et al. Effects of condom social marketing on condom use in developing countries: a systematic review and meta-analysis, 1990-2010.Bull World Health Organ. 2012; 90(8): 613-22.

Artigo apresentado em 30/05/15 Artigo aprovado em 18/03/16 Artigo publicado no sistema em 31/03/16 